\title{
The Role of Intracellular Calcium Signaling in Hematopoietic Stem Cells
}

\author{
Grahn THM* \\ Division of Molecular Medicine and Gene Therapy, Lund University Hospital, Sweden \\ *Correspondence Author: Tan Hooi Min Grahn, Division of Molecular Medicine and \\ Gene Therapy, Lund Stem Cell Center, Lund University Hospital, Sweden; Email: \\ hooi_min.tan_grahn@med.lu.se
}

\section{Mini Review \\ Volume 3 Issue 1}

Received Date: December 13, 2019

Published Date: December 24, 2019

DOI: $10.23880 /$ ggtij-16000113

\begin{abstract}
Hematopoietic stem cells (HSCs) are perceived to reside at the top of a hierarchy in which HSCs possess self-renewal capacity and can progressively give rise to all blood lineage cells. Many findings consider calcium signalling as an extrinsic factor and a handful of studies show the direct role of calcium sensor in HSC regulation. We discuss how intracellular calcium $\left(\mathrm{Ca}_{\mathrm{i}}{ }^{2}+\right)$ pathway regulates and maintains $\mathrm{HSC}$, from the aspect of relevant calcium sensor proteins. In the future, with a combination of advances in single cell technologies, low-input proteomic platform and spatialimaging will be hopeful approaches towards identifying the mechanism of $\mathrm{Ca}_{\mathrm{i}}{ }^{2+}$ in $\mathrm{HSC}$ maintenance.
\end{abstract}

Keywords: Calcium signalling; Bone marrow; Hematopoietic

Abbreviations: HSCs: Hematopoietic Stem Cells; BM: Bone Marrow; HOX: Homeobox Domain;Mll: Mixed Lineage Leukemia; HSPCs: Hematopoietic Stem and Progenitors; SCF: Stem Cell Factor; CaM: Calmodulin; CaMKIV: Calmodulin-Dependent Protein Kinase IV; LSK: Lin-Sca-1+Kit+; CaMKKII: Calmodulin-Dependent Protein Kinase Kinase II; HSPC: Hematopoietic Stem and Progenitor Cell; PML: Promyelocytic Leukemia; PPAR$\delta$ : Proliferator-Activated Receptor $\delta$.

\section{Introduction}

Hematopoiesis is the process of blood formation that is occurred in the adult bone marrow (BM) cells. Blood is one of the most regenerative tissues and is capable to produce approximately one trillion new cells daily [1]. The persistence production on high demands of new blood cells is crucially dependent on a rare population of cells, namely the hematopoietic stem cells (HSCs) consists of a heterogeneity group of primitive cells [2]. HSCs are renowned to balance their self-renewal capacity with their differentiation into committed blood cells in various blood lineages in a tightly regulated environment [3].

HSCs differentiation is regulated through their extrinsic factors, and the orchestrated activities of various intrinsic factors. To date, there are several revised models for hematopoietic stem cell differentiation [4]. Major intrinsic determinant of cell fate choices comprises of transcription factors, cell cycle regulators, anti-apoptotic signals and epigenetic factors. Briefly, transcription factors of the homeobox domain (HOX) family genes are important for HSC regulation [5]. 


\section{Genomics \& Gene Therapy International Journal}

Functional loss of the Hox co-factor MEIS1 increased HSC cycling and subsequent HSC exhaustion [6]. The deficiency of HoxB4 and HoxA9 leads to a reduction of HSC function [7]. Epigenetics modification controls the accessibility of target gene regulatory elements on core histones. The mixed lineage leukemia (Mll1) interacts with H3K4me3 histone modification and MLL1 loss has been linked to abnormal proliferation of hematopoietic stem and progenitors (HSPCs) and hematopoietic failure [8]. Besides, HSCs are suggested to reside in niches in the adult BM. For instance, stem cell factor (SCF) binds to KIT receptors, and deletion of SCF in endothelial and perivascular cells leads to a decrease in HSC numbers [9].

However, there is very little information available about the signal transduction events that regulate HSC function; in particular, the effects of intracellular calcium $\left(\mathrm{Ca}_{\mathrm{i}}{ }^{2+}\right)$ signaling are not well described. This review focuses on the participation of intracellular calcium and calcium binding protein in HSCs regulatory mechanisms.

Recent studies have added an important aspect of this process, introducing the role of $\mathrm{Ca}_{\mathrm{i}}{ }^{2+}$ in cell fate decisions during hematopoiesis. The balance between quiescence and divisions in HSCs is essential for hematopoietic maintenance. A study showed that the mechanism of how HSCs switch from quiescence to cycling during stress is governed through $\mathrm{Ca}_{i}{ }^{2+}$-mitochondria dependent pathway in the regulation of HSC division. The authors demonstrated that by suppressing the $\mathrm{Ca}_{\mathrm{i}}{ }^{2+}$ level using exogenous adenosine or Nifedipine, a $\mathrm{Ca}^{2+}$ channel blocker could prolong cell division interval in HSCs [10]. Besides, another current finding emphasized that how in vitro culture condition with low calcium could inhibit calpain proteases and differentiation, thereby improving on HSC maintenance in vitro [11]. These two studies however invite some debates regarding the exact role of $\mathrm{Ca}_{\mathrm{i}}{ }^{2+}$ in regulating the HSC.

Calmodulin (CaM) is an $\mathrm{Ca}^{2+}$ sensor and it binds to free cytosolic $\mathrm{Ca}^{2+}$, which leads to conformational changes that facilitate its interaction with the multifunctional $\mathrm{Ca}^{2+} / \mathrm{CaM}$-dependent protein [12]. Calmodulin-dependent protein kinase IV (CaMKIV), a downstream kinase of calmodulin is required for Lin-Sca- $1+\mathrm{Kit}^{+}$(LSK) cells to repopulate the bone marrow in transplantation assays and the role of CaMKIV in HSC self-renewal is mediated via regulation of $\mathrm{CBP}$ and $\mathrm{Bcl}-2$. The caveat of this finding is the authors defined the HSC by LSK surface markers, in which this population includes many early progenitors and primitive lymphoid cells.
Another more recent finding demonstrated that calmodulin-dependent protein kinase kinase II (CaMKKII) regulates the regeneration of hematopoietic stem and progenitor cells (HSPC). They suggested CaMKK2 links proximal radiation signaling to activation of the antiproliferative AMPK/p53 signaling pathways [13]. The authors have applied two sets of surface markers to define HSC population, LSK in the microarray while LSK CD34- in the transplantation setting, where raises thequestions of the quiescence status from the microarray may not reflect the highly proliferative phenotype that they have observed after the radiation.

Altogether, the findings that have been discussed above indicate that $\mathrm{Ca}_{\mathrm{i}}{ }^{2+}$ levels are crucial in HSC regulation that definitely requires more thorough investigation especially at the protein level, within the scope on other unknown $\mathrm{Ca}^{2+}$ sensors in determining HSC cell fate options. Quiescent HSCs is also featured for their low baseline energy production and low mitochondria membrane potentials and rely on glycolysis [14,15]. Recently, the effect on how metabolism regulates HSC function has received a great amount of attention, a role previously overlooked due to HSCs being thought of as mostly glycolytic. In addition, HSC maintenance has been linked to fatty acid oxidation through the promyelocyticleukemia (PML)-peroxisome proliferatoractivated receptor $\delta$ (PPAR- $\delta$ ) pathway as an alternative source of energy [16]. Advanced technologies have broadened our knowledge on hematopoiesis.

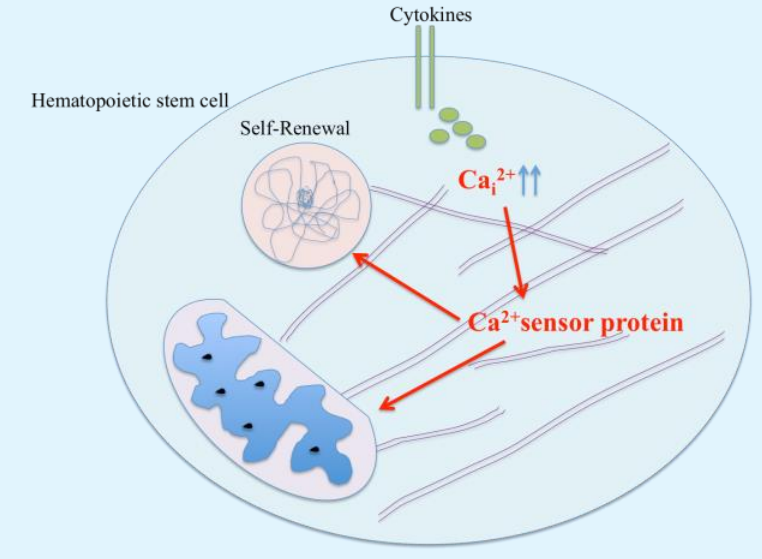

Figure 1: Schematic illustrates the Essential Role of Calcium Sensing Protein in HSC Regulation. Round pinkish: nucleus; rectangular: mitochondria; Elongated filament: actin filament. 


\section{Genomics \& Gene Therapy International Journal}

Hematopoietic stem cell fate option is regulated in a complex networks, from transcriptional to posttranscriptional, and even at the protein level. In consideration of all the studies discussed above, the model shown in Figure 1 represents the missing nodes. However, we believe that with the advances in single cell technology and proteomic platform that accepts low input of cells, more missing nodes of stem and progenitor cells mechanism will be discovered. Moreover, the epigenetic status of single HSPCs analyzed by single cell $\mathrm{Hi}-\mathrm{C}$ [17] and single cell ATAC-seq [18] provides information of the cell variability and the continuous regulatory landscape of HSC. By combining imaging with in situ RNA-seq $[19,20]$, studying the spatial localization of individual HSCs in combination with different transcriptomes becomes possible.

\section{References}

1. Ogawa M (1993) Differentiation and proliferation of hematopoietic stem cells. Blood 81(11): 2844-2853.

2. Morrison SJ, Uchida N, Weissman IL (1995) The biology of hematopoietic stem cells. Annu Rev Cell Dev Biol 11: 35-71.

3. Orkin SH (2000) Diversification of haematopoietic stem cells to specific lineages. Nat Rev Genet 1(1): 5764.

4. Cheng H, Zheng Z, Cheng T (2019) New paradigms on hematopoietic stem cell differentiation. Protein Cell.

5. Antonchuk J, Sauvageau G, Humphries RK (2002) HOXB4-induced expansion of adult hematopoietic stem cells ex vivo. Cell 109(1): 39-45.

6. Bijl J, Thompson A, Ramirez-Solis R, Krosl J, Grier DG, et al. (2006) Analysis of HSC activity and compensatory Hox gene expression profile in Hoxb cluster mutant fetal liver cells. Blood 108(1): 116122.

7. Brun $\mathrm{AC}$, Bjornsson JM, Magnusson $\mathrm{M}$, Larsson $\mathrm{N}$, Leveen P, et al. (2004) Hoxb4-deficient mice undergo normal hematopoietic development but exhibit a mild proliferation defect in hematopoietic stem cells. Blood 103(11): 4126-4133.

8. Jude CD, Climer L, Xu D, Artinger E, Fisher JK, et al. (2007) Unique and independent roles for MLL in adult hematopoietic stem cells and progenitors. Cell Stem Cell 1(3): 324-337.
9. Ding L, Saunders TL, Enikolopov G, Morrison SJ (2012) Endothelial and perivascular cells maintain haematopoietic stem cells. Nature 481(7382): 457462.

10. Umemoto $T$, Hashimoto $M$, Matsumura $T$, Nakamura Ishizu A, Suda T (2018) Ca(2+)-mitochondria axis drives cell division in hematopoietic stem cells. J Exp Med 215(8): 2097-2113.

11. Luchsinger LL, Strikoudis A, Danzl NM, Bush EC, Finlayson MO, et al. (2019) Harnessing Hematopoietic Stem Cell Low Intracellular Calcium Improves Their Maintenance In Vitro. Cell Stem Cell 25(2): 225-240.

12. Means AR (2008) The Year in Basic Science: calmodulin kinase cascades. MolEndocrinol 22(12): 2759-2765.

13. Racioppi L, Lento W, Huang W, Arvai S, Doan PL, et al. (2017) Calcium/calmodulin-dependent kinase kinase 2 regulates hematopoietic stem and progenitor cell regeneration. Cell Death Dis 8(10): 3076.

14. Suda T, Takubo K, Semenza GL (2011) Metabolic regulation of hematopoietic stem cells in the hypoxic niche. Cell Stem Cell 9(4): 298-310.

15. Ito K, Suda T (2014) Metabolic requirements for the maintenance of self- renewing stem cells. Nat Rev Mol Cell Biol 15(4): 243-256.

16. Ito $\mathrm{K}$, Carracedo A, Weiss $\mathrm{D}$, Arai $\mathrm{F}$, Ala U, et al. (2012). A PML-PPAR-delta pathway for fatty acid oxidation regulates hematopoietic stem cell maintenance. Nat Med 18(9): 1350-1358.

17. Nagano T, Lubling $Y$, Stevens TJ, Schoenfelder S, Yaffe E, et al. (2013) Single-cell Hi-C reveals cell-to-cell variability in chromosome structure. Nature 502(7469): 59-64.

18. Buenrostro JD, Corces MR, Lareau CA, Wu B, Schep AN, et al. (2018) Integrated Single-Cell Analysis Maps the Continuous Regulatory Landscape of Human Hematopoietic Differentiation. Cell 173(6): 15351548.

19. Wang X, Allen WE, Wright MA, Sylwestrak EL, Samusik N, et al. (2018) Three-dimensional intacttissue sequencing of single-cell transcriptional states. Science 361(6400). 


\section{Genomics \& Gene Therapy International Journal}

20. Eng CL, Lawson M, Zhu Q Dries R, Koulena N, et al. (2019) Transcriptome-scale super-resolved imaging in tissues by RNA seq FISH. Nature 568(7751): 235239. 